\title{
Clinical and radiological outcomes following traumatic Grade 3 and 4 vertebral artery injuries: a 10 -year retrospective analysis from a Level I trauma center. The Parkland Carotid and Vertebral Artery Injury Survey
}

\author{
William W. Scott, MD, ${ }^{1}$ Steven Sharp, MD, ${ }^{1}$ Stephen A. Figueroa, MD, ${ }^{2}$ \\ Alexander L. Eastman, MD, MPH, ${ }^{3}$ Charles V. Hatchette, MD, ${ }^{1}$ Christopher J. Madden, MD, ${ }^{1}$ \\ and Kim L. Rickert, MD ${ }^{1}$
} Departments of ${ }^{1}$ Neurosurgery, ${ }^{2}$ Neurocritical Care, and ${ }^{3}$ Trauma Surgery, University of Texas Southwestern Medical Center,
Dallas, Texas

\begin{abstract}
OBJECT Grade 3 and 4 blunt vertebral artery (VA) injuries may carry a different natural course from that of lower-grade blunt VA injuries. Proper screening, management, and follow-up of these injuries remain controversial. Grade 3 and 4 blunt VA injuries were analyzed to define their natural history and establish a rational management plan based on lesion progression and cerebral infarction.
\end{abstract}

METHODS A retrospective review of a prospectively maintained database of all blunt traumatic carotid and vertebral artery injuries from August 2003 to April 2013 was performed, and Grade 3 and 4 blunt VA injuries were identified. Grade 3 injuries were defined as stenosis of the vessel greater than $50 \%$ or the development of a pseudoaneurysm, and Grade 4 injuries were defined as complete vessel occlusion. Demographic information, radiographic imaging findings, number of imaging sessions performed per individual, length of radiographic follow-up, radiographic outcome at end of follow-up, treatment(s) provided, and documentation of ischemic stroke or transient ischemic attack were recorded.

RESULTS A total of 79 high-grade (Grade 3 and 4) blunt VA injuries in 67 patients were identified. Fifty-nine patients with 66 high-grade blunt VA injuries were available for follow-up. There were 17 patients with 23 Grade 3 injuries and 42 patients with 43 Grade 4 injuries. The mean follow-up duration was 58 days for Grade 3 and 67 days for Grade 4 blunt VA injuries. Repeat imaging of Grade 3 blunt VA injuries showed that $39 \%$ of injuries were radiographically stable, $43 \%$ resolved, and 13\% improved, while 1 injury radiographically worsened. Repeat imaging of the Grade 4 blunt VA injuries showed that $65 \%$ of injuries were radiographically stable (persistent occlusion), $30 \%$ improved (recanalization of the vessel), and in 2 cases (5\%) the injury resolved. All Grade 3 injuries that were treated were managed with aspirin or clopidogrel alone, as were the majority of Grade 4 injuries. There were 3 cerebral infarctions thought to be related to Grade 4 blunt VA injuries, which were likely present on admission. All 3 of these patients died at a mean of 13.7 days after hospital admission. No cerebral infarctions directly related to Grade 3 blunt VA injuries were identified.

CONCLUSIONS The majority of high-grade blunt VA injuries remain stable or are improved at final follow-up. Despite a 4\% rate of radiographic worsening in the Grade 3 blunt VA injury group and a 35\% recanalization rate in the Grade 4 blunt VA injury group, there were no adverse clinical outcomes associated with these radiographic changes. No cerebral infarctions were noted in the Grade 3 group. A 7\% stroke rate was identified in the Grade 4 blunt VA injury group; however, this was confined to the immediate postinjury period and was associated with $100 \%$ mortality. While these data suggest that these high-grade vertebral artery injuries may require less intensive radiographic follow-up, future prospective studies are needed to make conclusive changes related to treatment and management.

http://thejns.org/doi/abs/10.3171/2014.9.JNS1461

KEY WORDS blunt cervical vascular injury; vertebral artery injury; cerebral infarction; vascular disorders; trauma

ABBREVIATIONS CA = carotid artery; CTA = CT angiography; DSA = digital subtraction angiography; GCS = Glasgow Coma Scale; MVC = motor vehicle collision; PCVAIS = Parkland Carotid and Vertebral Artery Injury Survey; VA = vertebral artery.

SUBMITTED January 9, 2014. ACCEPTED September 2, 2014.

INCLUDE WHEN CITING Published online October 24, 2014; DOI: 10.3171/2014.9.JNS1461.

DISCLOSURE The authors report no conflict of interest concerning the materials or methods used in this study or the findings specified in this paper. 
$\mathrm{G}$ RADE 3 and 4 vascular injuries are believed to behave differently from Grade 1 and 2 injuries and are less likely to resolve. ${ }^{4,5}$ Management of these injuries remains heavily debated, but antiplatelet or anticoagulant medications tend to be first-line treatments. Endovascular stenting is usually reserved for lesions that show progression or severe stenosis causing neurological deficit. $1,4,7,8,15$ While our earlier report explored Grade 1 and 2 blunt vertebral artery (VA) injuries, this current paper evaluates Grade 3 and 4 blunt VA injuries. ${ }^{14}$ Imaging findings, progression of injury, and outcomes of these higher-grade blunt VA injuries were analyzed to gain a better understanding of their natural history to assist in establishing a rational management plan based on their progression and risk of infarct.

\section{Methods}

A retrospective review of a prospectively maintained database of all traumatic carotid artery (CA) and vertebral artery (VA) injuries was conducted at our Level I Trauma Center (Parkland Memorial Hospital). Attention was focused on Grade 3 and 4 blunt VA injuries. Since we suspect that higher-grade injuries may carry the potential to behave differently compared with lower-grade injuries, our aim for the Parkland Carotid and Vertebral Artery Injury Survey (PCVAIS) was to categorize and report on these injuries separately.

Screening for blunt cerebrovascular injuries at our institution occurs through a modification of the Denver Criteria. ${ }^{3}$ Any patient found to have cervical spine fractures of the vertebral body or pedicle, basilar skull fractures involving the carotid canal or petrous bone, LeFort II or III fractures, Glasgow Coma Scale (GCS) score lower than 7 without obvious cerebral injury on head CT, and/or near hanging/strangulation with a ligature mark/contusion undergoes screening cervical CT angiography (CTA) to rule out a vascular injury.

Grade 1 injuries are defined as a vessel lumen stenosis of less than 25\%, and Grade 2 injuries are defined as a stenosis of the vessel lumen between $25 \%$ and $50 \%$. We define Grade 3 injuries as stenosis of the vessel greater than $50 \%$, or the development of a pseudoaneurysm, and we define Grade 4 injuries as vessel occlusion. ${ }^{3}$ Grade 5 injuries are complete transections of the artery. All vascular injuries were identified by our Department of Neuroradiology. A staff neurosurgeon confirmed all injuries and decided on the desired follow-up and treatment. Only blunt injuries were evaluated. Any vascular injuries due to penetrating trauma were not included in this analysis.

Data including age, sex, mechanism of injury, presence or absence of a cervical fracture, and other traumatic injuries within the proximity of the neck (for example, occipital condyle fracture and first rib fracture) were recorded. Radiographic imaging was reviewed (for example, CT angiography [CTA], digital subtraction angiography [DSA]), as were the number of imaging sessions performed per individual and length of time between imaging and radiographic outcomes at the end of follow-up. Treatment provided was recorded. The presence of cerebral infarction was confirmed by clinical presentation and neuroimaging.

\section{Results}

From August 2003 through April 2013, there were 537 blunt cervical vascular injuries. This included $241(45 \%)$ CA and 296 (55\%) VA injuries. From this cohort, there were 79 Grade 3 or 4 blunt VA injuries in 67 patients. This comprised $13 \%$ of all blunt cervical vascular injuries and $27 \%$ of all VA injuries. Of these 67 patients, 59 were available for follow-up and were analyzed further in this study. There were 23 Grade 3 and 43 Grade 4 blunt VA injuries. There were 7 left, 4 right, and 6 bilateral Grade 3 blunt VA injuries and 23 left, 18 right, and 1 bilateral Grade 4 blunt VA injuries. Seven (41\%) of the Grade 3 cases and $28(67 \%)$ of the Grade 4 cases involved male patients. The mean patient age for Grade 3 injuries was 42 years (range $20-76$ years) and 48 years (range 17-93 years) for Grade 4 injuries.

Of the 8 patients who were excluded, 2 individuals, with a mean age of 29 years, presented dead on arrival after motor vehicle collision (MVC) with multisystem trauma that included left-sided Grade 4 blunt VA injuries (present in both cases) and cervical spine injuries consisting of occipital-cervical distraction and midcervical fracture subluxation. Three patients, with a mean age of 56 years, died shortly after arrival due to the magnitude of their multisystem trauma that included single-sided Grade 4 blunt VA injuries (2 left and 1 right), severe closed head injuries, cervical fracture-subluxations, and spinal cord injuries. Three patients, with a mean age of 76 years, underwent follow-up in our clinic for an average of 2.7 months for their cervical spine injuries but did not undergo reimaging with CTA due to their advanced age, comorbidities, and overall stable neurological condition. These 3 individuals all had Grade 4 blunt VA injuries (1 left, 1 right, and 1 bilateral), and were all treated indefinitely with antiplatelet medication for their vascular injury ( 2 with aspirin and 1 with aspirin and clopidogrel).

The mechanism of injury of Grade 3 injuries was MVC in 10 patients (64\%), fall in 2 patients (12\%), and motorcycle collision in 1 patient $(6 \%)$. Other less common mechanisms included assault, all-terrain vehicle accidents, and motor-pedestrian collisions in the remaining 4 patients $(24 \%)$. The mechanism of injury of Grade 4 injuries was MVC in 27 patients (59\%), fall in 10 patients $(24 \%)$, and motorcycle collision in 3 patients $(7 \%)$. Other less common mechanisms included assault, all-terrain vehicle accidents, and motor-pedestrian collisions in the remaining 2 patients $(5 \%)$.

\section{Grade 3 Injuries}

Of the Grade 3 injuries, 7 patients (41\%) had a singlesided Grade 3 blunt VA injury, while 10 patients $(59 \%)$ presented with multiple cervical vascular injuries (an additional ipsilateral and/or contralateral blunt VA injury and/or a blunt CA injury). The distribution of other vascular injuries is shown in Table 1. Cervical fractures were present in 9 patients (53\%), and of those with a cervical fracture, the transverse foramen was involved in $33 \%$.

Repeat imaging of the 23 Grade 3 blunt VA injuries ultimately demonstrated that 9 injuries $(39 \%)$ were stable, 10 injuries (43\%) were resolved, 3 injuries (13\%) were im- 
TABLE 1. Distribution of additional blunt VA injuries and blunt CA injuries in relation to Grade 3 blunt VA injury

\begin{tabular}{cccccc}
\hline & \multicolumn{2}{c}{ VA Injury } & & \multicolumn{2}{c}{ CA Injury } \\
\cline { 2 - 3 } \cline { 5 - 6 } Grade & Ipsilateral & Contralateral & & Ipsilateral & Contralateral \\
\hline 1 & 0 & 0 & & 2 & 1 \\
\hline 2 & 2 & 0 & & 0 & 0 \\
\hline 3 & 0 & 6 & & 3 & 0 \\
\hline 4 & 0 & 0 & & 0 & 0 \\
\hline
\end{tabular}

proved, and 1 injury (4\%) worsened (Table 2). In the 1 case of injury worsening (4\% of the Grade 3 group) there was increased stenosis at the injury on the first follow-up imaging. This injury was asymptomatic and did not progress to a Grade 4 injury.

During follow-up, 8 individuals (47\%) underwent only 1 follow-up CTA session (2 CT angiograms total). Nine patients underwent more than 2 CTA sessions: 4 patients had 3 sessions, 3 patients had 4 sessions, and 1 patient had 5 sessions. In $75 \%$ of cases, a follow-up CTA image was obtained within 7-10 days. During this short timeframe, $23 \%$ of the cases were noted to have injury resolution, $62 \%$ remained stable, 1 patient had improved to a Grade 1 injury, and 1 patient had radiographically worsened (further narrowing of the vessel without progressing to a Grade 4 injury). The mean duration of follow-up in the Grade 3 blunt VA injury group was 58 days (range 7-180 days). CTA was used as the initial imaging modality in all Grade 3 blunt VA injury cases.

All patients with Grade 3 blunt VA injuries in this study who were treated received either aspirin or clopidogrel (14 received aspirin and 2 received clopidogrel). One individual was not treated with a postinjury medication. This patient was a 76-year-old woman who demonstrated radiographic stability of her injury on follow-up;

TABLE 2. Progression and treatment of Grade 3 and 4 blunt VA injuries

\begin{tabular}{lcc}
\hline \multicolumn{1}{c}{ Variable } & Grade 3 & Grade 4 \\
\hline Progression* & & \\
\hline Stable & $39 \%$ & $65 \%$ \\
\hline Improved & $13 \%$ & $30 \%$ \\
\hline Resolved & $43 \%$ & $5 \%$ \\
\hline Worsened & $4 \%$ & $0 \%$ \\
\hline Follow-up duration in days (range) & $58(7-180)$ & $67(7-240)$ \\
\hline Treatment† & & \\
\hline Aspirin & $82 \% \ddagger$ & $64 \%$ \\
\hline Clopidogrel & $12 \%$ & $5 \%$ \\
\hline Endovascular embolization & $0 \%$ & $26 \% \S$ \\
\hline Heparin/Coumadin & $0 \%$ & $5 \%$ \\
\hline
\end{tabular}

* Percentages are based on the number of injuries.

$\dagger$ Percentages are based on the number of patients.

$\ddagger$ All except one patient in the Grade 3 blunt VA injury group was treated with postinjury antiplatelet medication.

$\S$ All except one patient who underwent endovascular embolization was also treated with a postprocedure antiplatelet medication. however, antiplatelet or anticoagulant medication in this case was thought precluded due to additional traumatic injuries. One individual who was identified as having a cerebral infarction (discussed below) was treated initially with heparin and Coumadin and later transitioned to aspirin. In all cases in which clinic follow-up records were available and showed resolution of the vascular injury, the treatment medication was discontinued. In those cases in which the injury remained present at the last available follow-up record, the treatment medication was recommended to be continued.

Magnetic resonance imaging was performed in 6 patients for evaluation of trauma or suspicion of stroke. One patient was found to have bilateral frontoparietal cerebral infarctions. The infarction pattern was thought to be related to concurrent bilateral CA injuries. This patient was a 44-year-old woman who was involved in an MVC, sustained multiple orthopedic injuries including a $\mathrm{Cl}-2$ dislocation with rotatory subluxation, and was identified as having a right-sided Grade 3 blunt VA injury in addition to bilateral Grade $2 \mathrm{CA}$ injuries. The Grade 3 blunt VA injury resolved on follow-up imaging (at 3 weeks, third CTA imaging session). This patient developed altered mental status on hospital Day 2 and MRI was performed, which demonstrated scattered, bilateral frontal and parietal subcortical hemispheric infarcts. She was treated initially with heparin and later transitioned to aspirin for her vascular injury. The patient was discharged to inpatient rehabilitation on hospital Day 32.

\section{Grade 4 Injuries}

Forty-three Grade 4 blunt VA injuries were identified. Thirty-four patients (81\%) had a single-sided Grade 4 blunt VA injury, while 8 patients (19\%) presented with multiple cervical vascular injuries (an additional ipsilateral and/or contralateral blunt VA injury and/or a blunt CA injury). The distribution of other vascular injuries is shown in Table 3. Cervical fractures were present in 39 patients (93\%), and of those with a cervical fracture, the transverse foramen was involved in $29 \%$.

Repeat imaging of the Grade 4 blunt VA injuries ultimately demonstrated that 28 injuries $(65 \%)$ were stable (persistent occlusion), 13 injuries (30\%) were improved (recanalization of the vessel, with 7 injuries improving to a Grade 1 injury), and in 2 cases (5\%) the injury was found to have resolved at final follow-up (Table 2). Twenty-seven patients (64\%) underwent imaging with CTA only, 14 patients (33\%) underwent imaging using both CTA and DSA, and 1 patient underwent imaging only with DSA. The first follow-up image was obtained in the majority

TABLE 3. Distribution of additional blunt VA injuries and blunt $\mathrm{CA}$ injuries in relation to Grade 4 blunt VA injury

\begin{tabular}{cccccc}
\hline & \multicolumn{2}{c}{ VA Injury } & & \multicolumn{2}{c}{ CA Injury } \\
\cline { 2 - 3 } \cline { 5 - 6 } Grade & Ipsilateral & Contralateral & & Ipsilateral & Contralateral \\
\hline 1 & 0 & 0 & & 4 & 1 \\
\hline 2 & 0 & 0 & 0 & 0 \\
\hline 3 & 0 & 0 & 2 & 2 \\
\hline 4 & 0 & 1 & 0 & 0 \\
\hline
\end{tabular}


of patients in this cohort (83\%) within 7-10 days. During this short timeframe, these Grade 4 injuries were identified as stable in $91 \%$ of cases, and 2 (9\%) improved to Grade 1 injuries. The mean duration of follow-up in this Grade 4 injury cohort was 67 days (range 7-240 days).

Grade 4 injuries were managed with aspirin or clopidogrel alone in 29 patients $(27$ received aspirin and 2 received clopidogrel), and 2 cases were treated with heparin and Coumadin. Eleven patients (26\%) underwent coil embolization of the affected VA, with the majority of those embolizations (7 patients, 64\%) being performed preemptively within the first few days after injury for concern of embolic stroke in the event of possible vessel recanalization. Two patients underwent coil embolization for asymptomatic vessel recanalization noted on the third and fourth follow-up CTA sessions, respectively. One patient underwent embolization because of development of a pseudoaneurysm proximal to a stable occlusion, and 1 patient underwent embolization because he was not deemed a candidate for anticoagulation due to a concomitant liver injury. All patients but one who underwent endovascular embolization were also treated with a postprocedure antiplatelet medication. In the individual who did not receive postprocedure medication, the reason was coagulopathy related to liver trauma. All cases of angiography with subsequent embolization occurred prior to 2009 , with the majority of these cases occurring between 2004 and 2007. In all cases in which clinic follow-up records were available and there was resolution of the vascular injury, the treatment medication was discontinued. In those cases in which the injury remained present at the last available follow-up record, it was recommended that the treatment medication be continued.

Seven patients (17\% of the Grade 4 blunt VA injury group) underwent MRI for trauma evaluation or suspicion of stroke. Three patients (7\% of the Grade 4 blunt VA injury group) had posttraumatic cerebral infarctions that were thought to be related to the Grade 4 blunt VA injury. One of these patients (33\% of this infarct group) had bilateral Grade 4 blunt VA injuries. The mean timeframe for obtaining an MRI in these cases was 2.3 days (range 1-3 days). The mortality rate of these infarct cases was $100 \%$, with a mean time to death of 13.7 days after hospital admission (range 2-33 days). The mean GCS score on arrival to the hospital in this infarction group was 3.7 (range $3-5$ ). It is our contention that these strokes were present on or soon after hospital admission. The first patient was a 44-year-old woman who was involved in an MVC and presented to our trauma center with a GCS score of 5. She had no cervical or major orthopedic injuries. CTA of the head and neck demonstrated a left-sided Grade 4 blunt VA injury. Her examination did not improve after admission, and on hospital Day 3, brain MRI was performed, which demonstrated multiple areas of acute ischemic changes in the bilateral occipital lobes and the posterior corpus callosum. The decision was made to withdraw further medical care, and the patient died on hospital Day 33. The second patient was a 45-year-old woman who was involved in an MVC and presented with a GCS score of 3. She had sustained multiple orthopedic injuries, including multiple skull base fractures. Her workup was significant for a right-sided Grade 4 blunt VA injury as well as an aorta injury. Her neurological examination did not improve after admission, and on hospital Day 3 MRI of the brain was performed and demonstrated bilateral posterior cerebral artery infarctions. Heparin was initiated; however, shortly thereafter, medical care was withdrawn and she died on hospital Day 6. The third patient was a 79-year-old man who had sustained a fall. He suffered multiple orthopedic injuries, including a $\mathrm{C} 2-3$ bilateral subluxation. $\mathrm{He}$ was also identified as having bilateral Grade 4 blunt VA injuries in addition to an occluded basilar artery. Due to his poor neurological status and the CTA findings, MRI of the brain was performed, which demonstrated bilateral cerebellar, left occipital, and thalamic infarcts. No strokerelated medical treatments were initiated in this case and he died on hospital Day 2.

\section{Discussion}

To avoid redundancy from our earlier report on lowgrade blunt VA injuries, the discussion here is limited to our experience with higher-grade VA injuries. ${ }^{14}$ Grade 3 and 4 vascular injuries are believed to behave differently from Grade 1 and 2 injuries and are less likely to resolve., ${ }^{4,5}$ In this series of high-grade blunt VA injuries, while there was a $4 \%$ rate of radiographic worsening, $42 \%$ of Grade 3 blunt VA injuries remained stable, and an equal number of these injuries resolved. The majority of the Grade 4 blunt VA injuries remained stable $(65 \%$ demonstrated persistent occlusion at final follow-up); however, $35 \%$ demonstrated recanalization of the vessel on follow-up imaging (50\% of these recanalization cases improved to a Grade 1 injury), while 5\% demonstrated injury resolution.

Our study identified 4 cases of ischemic stroke, as determined by posttraumatic neuroimaging. The cerebral infarction identified in the patient with a Grade 3 blunt VA injury was felt to be related to concurrent bilateral blunt CA injuries, and was not examined further. The cases of stroke that were seen and were determined to be the result of the high-grade blunt VA injury were identified at a mean of 2.3 days after admission and were all a result of a Grade 4 blunt VA injury. Due to the severity of the clinical presentation in these cases, and rapid documentation of these injuries from the trauma bay, our contention is that these cerebral infarctions were likely present by the time of hospital arrival. Griessenauer et al. ${ }^{6}$ found similar conclusions, noting that most cerebral strokes occurred close to the time of injury. In the Biffl et al. series, ${ }^{4}$ in those individuals who suffered a posttraumatic stroke, $90 \%$ of infarctions were noted prior to any medical treatments, $9 \%$ were identified while individuals were on an aspirin regimen, and $1 \%$ occurred while the patients were on a heparin regimen. The majority of the strokes identified in our study were also found prior to starting any treatment. Those individuals with Grade 4 blunt VA injury who were identified as having posttraumatic infarction presented with a poorer overall GCS score; their strokes were identified soon after hospital admission and prior to any treatment; and it is possible that these cerebral infarctions were present on admission or shortly after. The occurrence of strokes in this population is clearly a marker 
for a severe level of injury. These patients had a $100 \%$ mortality rate.

Management of these injuries continues to remain heavily debated, but antiplatelet or anticoagulant medications tend to be first-line treatments, with endovascular stenting reserved for symptomatic or worsening stenosis. ${ }^{1,4,7,8,15}$ In the current study, while no endovascular intervention was performed in the Grade 3 blunt VA injury group, 11 individuals in the Grade 4 blunt VA injury group underwent coil embolization of the affected VA, predominantly in a preemptive manner. Those procedures occurred early in our experience (November 2004 to January 2008), and after 2008 we no longer performed coiling for occluded arteries. In 2009, Biffl et al. ${ }^{5}$ recommended against stenting for any grade blunt VA injury due to a higher associated morbidity than with medical treatment alone. We did identify one patient in 2005 who harbored a concomitant Grade 3 blunt CA injury in addition to a Grade 4 blunt VA injury. This patient underwent an unsuccessful stenting procedure for the internal carotid artery injury and was subsequently treated with clopidogrel. While no stent procedures were performed directly for VA injuries in this study, endovascular stent procedures for various blunt $\mathrm{CA}$ injuries during the timeframe examined in this study were performed, which will be discussed in upcoming reports. Since all but one patient included in the high-grade blunt VA injury study groups underwent medical treatment, we do not know if treatment alters the natural history of the disease. We do show that the cerebral infarctions identified in these highgrade blunt VA injury groups occurred soon after injury and prior to the administration of any medication.

Cervical fracture injuries were present in 53\% of the Grade 3 blunt VA injuries and in 93\% of Grade 4 blunt VA injuries with transverse foramen involvement in $33 \%$ and $29 \%$, respectively. High correlation between cervical fracture and blunt VA injury has also been established in previous papers. ${ }^{10,12,13}$ We did not find any other convincing correlation between musculoskeletal injuries in the proximity of the neck and rate of blunt VA injury.

While data procured for this study were collected through a prospective database, the major limitation of this study relates to its retrospective analysis. A randomized controlled trial of various treatment strategies is needed to verify appropriate follow-up protocols. Although some studies have suggested that CTA overestimates vascular injury in these blunt trauma cases, ${ }^{6,10,11,16}$ we used this imaging modality in the majority of cases due to the comparative ease, accessibility, and safety associated with this procedure compared with DSA, especially in critically ill, multitrauma patients. Use of CTA may have then produced a falsely elevated incidence rate of vascular injury at our center. Similarly, a falsely elevated rate of improvement on follow-up imaging may also be reflected in this study as compared with older studies in which DSA was used, ${ }^{2,9}$ especially if there was a potential overestimation of initial injury.

We experienced large variability in the length of follow-up time, which does limit our ability to make conclusions about the time course of healing of these highgrade VA injuries. Along the same lines, variation in treatment over the 10-year study period may have altered the clinical outcome in these cases. Another limitation of this retrospective study is that we cannot be entirely sure that some clinically silent strokes were not missed, but we can state that all individuals included in this study did undergo follow-up evaluations, and thus we are certain that all clinically apparent strokes were both identified and recorded. While these data across the past 10 years at our institution yielded valuable information, the data involving high-grade VA injuries alone did not afford us much ability for statistical analysis; however, once all data across all cervical vascular injuries have been analyzed, we look forward to the ability to offer a wellpowered analysis across all grades of blunt VA and CA injuries, specifically relating to the stroke risk in the setting of treatment, timing, vessel, and grade.

\section{Conclusions}

Results of this study suggest that Grade 3 blunt VA injuries carry a low stroke risk. Despite a 35\% recanalization rate, the Grade 4 blunt VA injuries have a benign clinical course in the long term; however, they do carry a $7 \%$ stroke rate confined to the immediate postinjury period, which was associated with $100 \%$ mortality. It is our contention that these infarctions were likely present on hospital admission. Antiplatelet and/or anticoagulant medication may be necessary in this high-grade blunt VA injury group; however, in the absence of infarctions after the postinjury period and the tendency for the majority of these injuries to remain stable or improve to a lowergrade injury, the long-term efficacy of antiplatelet medication is not yet entirely clear. The time and cost allotted for follow-up radiography deserves attention, as continued imaging in these subsets of vascular injury may not prove entirely necessary. Follow-up protocols should be amended, but further prospective studies are needed to make conclusive changes as it relates to management.

As we continue this PCVAIS study from our institution, we look forward to presenting our entire experience of cervical vascular injuries in sectioned categories as discussed above. After we conclude reporting on all grades of CA and VA injuries, we will at that time consider future plans for prospective research on this topic, specifically, determining which injuries warrant followup and which injuries require treatment.

\section{References}

1. Biffl WL, Cothren CC, Moore EE, Kozar R, Cocanour C, Davis JW, et al: Western Trauma Association critical decisions in trauma: screening for and treatment of blunt cerebrovascular injuries. J Trauma 67:1150-1153, 2009

2. Biffl WL, Moore EE, Elliott JP, Ray C, Offner PJ, Franciose RJ, et al: The devastating potential of blunt vertebral arterial injuries. Ann Surg 231:672-681, 2000

3. Biffl WL, Moore EE, Offner PJ, Brega KE, Franciose RJ, Burch JM: Blunt carotid arterial injuries: implications of a new grading scale. J Trauma 47:845-853, 1999

4. Biffl WL, Ray CE Jr, Moore EE, Franciose RJ, Aly S, Heyrosa MG, et al: Treatment-related outcomes from blunt cerebrovascular injuries: importance of routine follow-up arteriography. Ann Surg 235:699-707, 2002 
5. Cothren CC, Biffl WL, Moore EE, Kashuk JL, Johnson JL: Treatment for blunt cerebrovascular injuries: equivalence of anticoagulation and antiplatelet agents. Arch Surg 144:685690,2009

6. Griessenauer CJ, Fleming JB, Richards BF, Cava LP, Curé $\mathrm{JK}$, Younan DS, et al: Timing and mechanism of ischemic stroke due to extracranial blunt traumatic cerebrovascular injury. Clinical article. J Neurosurg 118:397-404, 2013

7. Kansagra AP, Cooke DL, English JD, Sincic RM, Amans MR, Dowd CF, et al: Current trends in endovascular management of traumatic cerebrovascular injury. J Neurointerv Surg 6:47-50, 2014

8. Li W, D'Ayala M, Hirshberg A, Briggs W, Wise L, Tortolani A: Comparison of conservative and operative treatment for blunt carotid injuries: analysis of the National Trauma Data Bank. J Vasc Surg 51:593-599, e1-e2, 2010

9. Miller PR, Fabian TC, Bee TK, Timmons S, Chamsuddin A, Finkle R, et al: Blunt cerebrovascular injuries: diagnosis and treatment. J Trauma 51:279-286, 2001

10. Miller PR, Fabian TC, Croce MA, Cagiannos C, Williams JS, Vang M, et al: Prospective screening for blunt cerebrovascular injuries: analysis of diagnostic modalities and outcomes. Ann Surg 236:386-395, 2002

11. Mutze S, Rademacher G, Matthes G, Hosten N, Stengel D: Blunt cerebrovascular injury in patients with blunt multiple trauma: diagnostic accuracy of duplex Doppler US and early CT angiography. Radiology 237:884-892, 2005

12. Ren X, Wang W, Zhang X, Pu Y, Wang M, Jiang T: The comparative study of magnetic resonance angiography diagnosis and pathology of blunt vertebral artery injury. Spine (Phila Pa 1976) 31:2124-2129, 2006
13. Schievink WI: Spontaneous dissection of the carotid and vertebral arteries. N Engl J Med 344:898-906, 2001

14. Scott WW, Sharp S, Figueroa SA, Madden CJ, Rickert KL: Clinical and radiological outcomes following traumatic Grade 1 and 2 vertebral artery injuries: a 10-year retrospective analysis from a Level 1 trauma center. Clinical article. J Neurosurg 121:450-456, 2014

15. Tardif JC, Cöté G, Lespérance J, Bourassa M, Lambert J, Doucet S, et al: Probucol and multivitamins in the prevention of restenosis after coronary angioplasty. N Engl J Med 337:365-372, 1997

16. Wang AC, Charters MA, Thawani JP, Than KD, Sullivan SE, Graziano GP: Evaluating the use and utility of noninvasive angiography in diagnosing traumatic blunt cerebrovascular injury. J Trauma Acute Care Surg 72:1601-1610, 2012

\section{Author Contributions}

Conception and design: Scott. Acquisition of data: Scott, Hatchette, Madden, Rickert. Analysis and interpretation of data: Scott, Sharp, Rickert. Drafting the article: Scott, Sharp. Critically revising the article: Scott, Figueroa, Eastman, Rickert. Reviewed submitted version of manuscript: all authors. Approved the final version of the manuscript on behalf of all authors: Scott. Study supervision: Rickert.

\section{Correspondence}

William W. Scott, UTSW Department of Neurosurgery, 5323 Harry Hines Blvd., Dallas, TX 75390-8855. email: william_w_ scott@hotmail.com. 